\title{
EDITORIAL Late diagnosis of HIV infection
}

In the mid-1990's, the expansion of the highly active antiretroviral therapy had an extraordinarily positive impact on the morbidity and mortality caused by the Human Immunodeficiency Virus (HIV) wherever treatment was provided to the infected population.

The countries which were most affected by the HIV pandemic lacked the necessary resources and infrastructure in order to make treatment accessible for all of those who needed it, so UNAIDS promoted the " 3 by 5 " initiative which aimed to achieve that by the year 2005 three million patients worldwide could have access to effective treatment.

Subsequently, in a historical turning point, governments committed to achieve universal access to HIV treatment by 2010 to all of those who needed it. To take stock of the progress and decide on future actions, a High-Level Meeting on AIDS will be held in New York City in June 2011.

Together with the lack of resources, the most important barrier for HIV infected patients to fully benefit from antiretroviral therapy is that a large number of them are unaware of their infection.

Developing countries account for the largest proportion of infected population who have not been diagnosed, nonetheless even in the European Union (EU) it is estimated that $30 \%$ of the people living with HIV remain undiagnosed. In addition to the deleterious effect it represents for patients, its impact on public health is devastating. In the United States, it has been estimated that $25 \%$ of the undiagnosed population is responsible for the transmission of new HIV infections in $54 \%$ of cases. On the other hand, late diagnosis of the infection involves the deterioration of the patient's immunity and makes treatment to the people in this situation enormously more expensive.

The serious individual and social repercussions that the undiagnosed fraction represents, have determined that in order to reduce it, the promotion of early diagnosis of the disease has become a priority.

Ideally, HIV infection should be diagnosed as soon as possible after transmission has taken place, nevertheless low specificity of HIV seroconversion and long- lasting asymptomatic period, even in the absence of treatment, make this process difficult.

In this context, it is considered that, as a minimum, diagnosis should be made before the patient's immunological status deteriorates enough as to require treatment; thus the most largely accepted definitions at present suggest that patients who have a CD 4 cell count below 350 cells/ $\mu$ l or an AIDS defining illness at the time of their HIV diagnosis should be defined as having a late diagnosis.

If late diagnosis is defined as a CD4 cell count below 350 cells $/ \mu$ at the time of HIV diagnosis, then $50.2 \%$ of new infections in Spain in 2009 corresponded to this situation. $30.4 \%$ of cases showed CD4 cell count below 200/ 1 l, that is to say that their immune system had suffered severe deterioration which could lead to the development of AIDS-defining diseases at any moment. Similar figures to those of our country were found in other EU countries where data was available ${ }^{2}$.

Distribution of late diagnosis is not random. Those affected by this situation in Spain, were mostly migrants, particularly those from Latin America and sub-Saharan Africa, individuals who contracted HIV through sharing injection equipment or heterosexual contact, individuals who are over 40 years old and males. Low incidence amongst women is attributed to antenatal HIV screening programs and can be observed in all the countries where they have been largely implemented.

What can be done in this situation? Any strategy to reduce late diagnosis must ensure that every individual can get tested if he wishes to. This requires testing to be free and accessible, and confidentiality 
as well as the patients' right must be guaranteed. However, even when those requirements are met, many do not get tested because they do not perceive risk, or fear the stigma attached to the illness.

This has led to promote policies which encourage health professionals to offer HIV testing, also known as "provider-initiated testing". More generally, there are two main strategies to carry out this initiative in countries with concentrated epidemics like Spain. The first one has been implemented in the United States in 2006 and supports that, when a certain threshold of prevalence has been reached - set there at $0.1 \%$ - from which the process is cost-effective, health professionals systematically offer HIV testing to anyone who requires health care and independently of the cause ${ }^{2}$. The second option supports that testing should not be generalized but targeted to sub-populations where probability of infection is higher, either because there is a higher incidence of risk behaviours - this is the case of prison inmates- or because there are signs/symptoms of AIDS defining or HIV related diseases such as tuberculosis, sexually transmitted diseases or hepatitis ${ }^{3}$. In the interest of feasibility, both strategies support the simplification of the testing process - generally the patient's consent and pre-test counselling - yet concrete proposals to carry out this simplification can vary considerably. Both option take into consideration generalized screening in pregnant women and mandatory HIV test in blood and organ donors.

Together with universal or selective access to testing from the health care professionals, policies to reduce late diagnosis normally consider other aspects such as frequency of HIV testing, implementation of community-based detection programs and access to rapid HIV testing.

Different international organizations and health care institutions of some countries recommend that certain populations get tested frequently; men who have sexual relations with other men should get tested every year in the absence of risk behaviours, whereas injection drug users should get tested between every six and twelve months.

Community-based programs for the detection of HIV aim to facilitate access to HIV testing to specific populations by offering tests outside of health care centres, pharmacies and community centres are among the most common places. In both cases, as well as in street outreach programs or in special events such as the gay pride, rapid HIV testing, whose sensitivity and specificity are very high, are essential for detection.

In December 2010, the European Centre for Disease Prevention and Control (ECDC) published a guide designed to inform the development, monitoring and evaluation of national HIV testing strategies in the countries of the European Union ${ }^{5}$. Any national strategy must be based on the following principles: a) HIV testing must be voluntary and confidential, with informed consent which should be documented but does not have to necessarily include the patient's signature; b) ensure access to treatment, care and prevention services; c) show political commitment, supported by financial investment and used in a cost-effective way; d) reduce stigma; e) remove legal and financial barriers, including mandatory testing policies - with the exception of blood and organ donations - and promote antidiscrimination legislation; f) develop and implement an HIV testing strategy with the participation of stakeholders; g) make access to HIV testing an integral part of all national health care strategies.

Within this last point, ECDC make special reference to health care in prison, pointing out that where HIV prevalence in prisons is known or estimated to be high, access to voluntary and confidential HIV testing as well as, wherever possible, screening for other sexually transmitted infections (STIs) and viral hepatitis should be an essential component of national programs aimed to increase access to HIV testing and reduce late diagnosis. Yet it must be said that the integration of prison health care within national HIV prevention strategies, including access to HIV testing, has been a reality in Spain for a long time and without a doubt has played an important role in achieving worldwide recognition.

\section{BIBLIOGRAPHIC REFERENCES}

1. ONUSIDA. Acceso universal a la prevención, tratamiento, atención y apoyo relacionados con el VIH: desde los países a las regiones hasta la reunión de alto nivel sobre sida y más allá. Hoja de Ruta 2011. Ginebra: ONUSIDA; 2011 [citado 2011 may 18]. Disponible en: http://www.unaids. 
org/en/media/unaids/contentassets/documents/ document/2011/2011_UA_roadmap_es.pdf.

2. European Centre for Disease Prevention and Control/WHO Regional Office for Europe. HIV/AIDS surveillance in Europe 2009. Stockholm: ECDC; 2010.

3. Branson BM, Handsfield HH, Lampe MA, Janssen RS, Taylor AW, Lyss SB, Clark JE, et al. Revised recommendations for HIV testing of adults, adolescents, and pregnant women in health-care settings. MMWR 2006; 55 (RR14): $1-17$.
4. UNAIDS/WHO. Guidance on providerinitiated HIV testing and counselling in health facilities. Geneva: UNAIDS/WHO; 2007

5. European Centre for Disease Prevention and Control. HIV Testing: increase uptake and effecttiveness in the European Union. Stockholm: ECDC; 2010.

\section{Mercedes Díez}

Unit of epidemiology of HIV and risk behaviours. Secretariat of the National Plan on AIDS. National Centre for Epidemiology mdiez@isciii.es 